\title{
CME
}

\section{List of CME}

SI

Department

1. $\quad$ "Fasting in Pregnancy" speaker: Prof. Shehereen F Siddiqua

May, 2018

2. "Role of Probiotics Biffidus Lactis to Ensure Long Term Health of C-Section Babies" June, 2018

Speaker: Prof. Shehereen F Siddiqua

3. "Infertility and Assisted Reproductive Technology" Organized by Department of

May, 2018

Infertility \& Reproductive Medicine

4. Seminar on "Upgrade from LDC Status \& Historic Achievement in Medical Sector" March, 2018 Organized by Anwer Khan Modern Medical College

5. CME on "SOE", organized by Medical Education Unit

6. $\quad$ CME on "SAQ \& MCQ", organized by Medical Education Unit April, 2018

7. CME on "Evidence Based Learning", organized by Medical Education Unit

June, 2018

\section{Workshop list}

SI

1. Live surgical workshop on "Breast Surgery" organized by Department of Surgery

\section{Department}

July, 2018 